\title{
Model and Simulation of Network Crisis Information Diffusion under Uncertain Environment
}

\author{
Yi-Rui Deng ${ }^{1}$ and Rui-Hong Zhang ${ }^{2}$ \\ ${ }^{1}$ School of Economics \& Management, China University of Petroleum, Qingdao 266555, China \\ ${ }^{2}$ National Science Library, Chinese Academy of Science, Beijing 100190, China
}

Correspondence should be addressed to Yi-Rui Deng; yiruideng@163.com

Received 14 July 2016; Accepted 7 December 2016

Academic Editor: Jan Weglarz

Copyright (C) 2016 Y.-R. Deng and R.-H. Zhang. This is an open access article distributed under the Creative Commons Attribution License, which permits unrestricted use, distribution, and reproduction in any medium, provided the original work is properly cited.

\begin{abstract}
Network crisis information diffusion will have a certain impact on the public's psychology and behavior and will cause harm to the normal operation social of the public system and the effective allocation of the public resources. So we should timely control the key factors which affect the diffusion process to reduce the damage. Using cellular automata theory, the paper views the public as a series of cellular automata and sets up some cellular state evolution rules. With the help of MATLAB simulated evolution, this paper explores the diffusion rule of crisis information diffusion process and finds out the key factors of crisis information diffusion process and its influence on the diffusion scale and effect, so as to put forward coping strategies. It is hoped that this paper provides reference for the theoretical study of the crisis information diffusion and provides suggestion for the real world to control the crisis information diffusion.
\end{abstract}

\section{Introduction}

Since the 21st century, natural disasters, the financial crisis, public health, social security, and other kinds of crisis events are staged all over the world. The spread and diffusion of crisis information from above crisis events have certain effect on public's psychology and behavior. Under adverse circumstances that information acquisition is not sufficient and the situation is uncertain, crisis information diffusion will make the core values of decision makers seriously threatened and challenged and will cause harm to the normal operation social of the public system and the effective allocation of the public resources. Also, crisis information diffusion will lead to interruption or paralysis of certain social activities and even affect human security and social stability.

Based on cellular automata theory (cellular automata, hereinafter referred to as $\mathrm{CA}$ ), this paper makes use of the BASS model in the field of new product or innovation diffusion to the process of crisis information diffusion and constructs the network crisis information diffusion system. Then this paper analyzes the diffusion motivation on the basis of system elements, determines the key factors, and constructs the transformation rules of crisis information diffusion state and studies its effect on the scale and effect of crisis information diffusion and analyzes the influence rules and effect factors of every factor. It is hoped that this paper improves the crisis information diffusion theory research and provides the corresponding countermeasures and measures of the crisis information diffusion control in the real world in order to reduce the risk of crisis information.

\section{Network Crisis Information Diffusion System}

2.1. Diffusion System. The network crisis information diffusion system is a complex integrated system composed of source, sink, crisis information, noise, diffusion media, and other elements, and it is a complex function system to realize the information diffusion between the source and the sink. The source and the sink exist in social network; they are the sender and receiver of crisis information. Crisis information attached to the critical incident is the main object of the diffusion process, and it contains the real and 
objective information and the false rumors. Noise is the information which has the interference function in the social environment. Diffusion media or channel is the way of crisis information diffusion, and different diffusion media will affect the effect of diffusion. Government agencies and public media are the third party that publishes real information and guides public opinion. They can spread the official authority information through various channels in the crisis information diffusion process, control the spread of rumors, and reduce the risk of rumors.

In this paper, the one meaning of network is that the network provides a rapid means of rapid diffusion to information diffusion. Mobile Internet provides a variety of convenient communication and makes all kinds of information diffusion stride across the limit of time and space and speed diffusion rapidly in a short time. The other meaning of network is the complex networks composed of the complex interpersonal relationships of individuals in reality [1]. It takes the individual in reality as a point, takes the link between the individual as a side, and makes use of side to connect the points. Then it will form a complex relationship network map.

2.2. Crisis Information Diffusion Motive. Diffusion motive is the source power that drives crisis information diffusing. From the perspective of origin, diffusion motive includes informational motive, emotional motive, and social motive [2].

2.2.1. Informational Motive. Informational motive is subdivided into government informational motive, media informational motive, and public informational motive. The purpose is to meet the needs of the public information. Responsibility drives news media and government departments to diffuse information. Public informational motive is an altruistic behavior in social psychology.

Government Informational Motive. The coordination function, control function, and supervisory function of the government require the government to ensure that people can effectively accomplish the administrative goal and guide and supervise the production and life of the society. So, in the crisis, the government must have a correct understanding of the crisis information and correct guidance.

Media Informational Motive. Information diffusion, knowledge promotion, and supervision of public opinion are the function of the media. The key function is that media should timely disseminate the real information, explore the typical and advanced things in real life, extensively and lastingly promotion knowledge, promote the exchange and innovation of knowledge, and then influence the public's mode of production and life. Also, media should adhere to the responsibilities of public opinion supervision, expose the social contradictions and evil, and reflect the fairness and justice.

Public Informational Motive. From the perspective of social psychology, public altruism is a kind of self-satisfaction. When the public is in panic, sadness, tension, and other negative feelings, helping behavior is conducive to eliminate the negative feelings of the heart, and the person in the crisis and the potential victims will have these negative feelings. Psychological experiments show that even if the helper does not receive in return, we will still help people who are in need of help. This is the embodiment of social responsibility and moral. Also this reflects that the public's own demand for information is the internal driving mechanism that the public get or learn about crisis information.

2.2.2. Emotional Motive. It is the motive that releasing crisis information is in order to give vent to emotion. After the crisis, the individual is prone to anxiety, panic, depression, and other negative emotions. At this time, the public can transfer his feel to acquaintances or strangers in the mobile terminal in order to weaken and vent their dissatisfaction.

2.2.3. Social Motive. It is the crisis information diffusion driven by social factors. Social motive includes seeking the public's help, winning the attention of others, and gaining the public recognition. When you are in trouble in a crisis, you can release information through the network and inform others and hope to get effective help. Winning the attention of others is that you can release crisis information to get more attention by curiosity when the crisis is not yet clear [3]. After the crisis, the public release information to gain recognition. That is to say, the so-called opinion leaders published their judgment and evaluation of the crisis, which can get the user's respect and praise.

2.3. Network Crisis Information Diffusion Process. In the early period of the crisis, the information is only in the hands of a small number of insiders. The large-scale diffusion through media is unlikely to happen. The entire diffusion network is in a calm state, and the information flow is very small.

With the evolution of time, people's demand for the crisis information is more and more, and crisis information owners' information will be released to the surrounding population through social networks. At the same time, government agencies, experts, scholars, and the media will also be involved, and the authoritative information is also released to the public. The amount of crisis information is great at this time and the information content is true or false. People will judge the type of information that is trusted according to the status of neighboring nodes and the amount of their information.

Then government agencies investigate and survey and take corresponding measures to control the situation, which makes the strength of the information flow of authoritative information increase. The crisis is gradually transparent, the deviation and error information are clarified, and the spread of rumors decreases. Public panic begins to eliminate, the need for information decreases, and the attention of the crisis is also slowly reduced. At this time, the amount of information is large, and the proportion of authoritative information content gradually increased.

Finally, the crisis has been fully resolved, and the social values and norms of behavior return to normal. The crisis information diffusion is ended. 


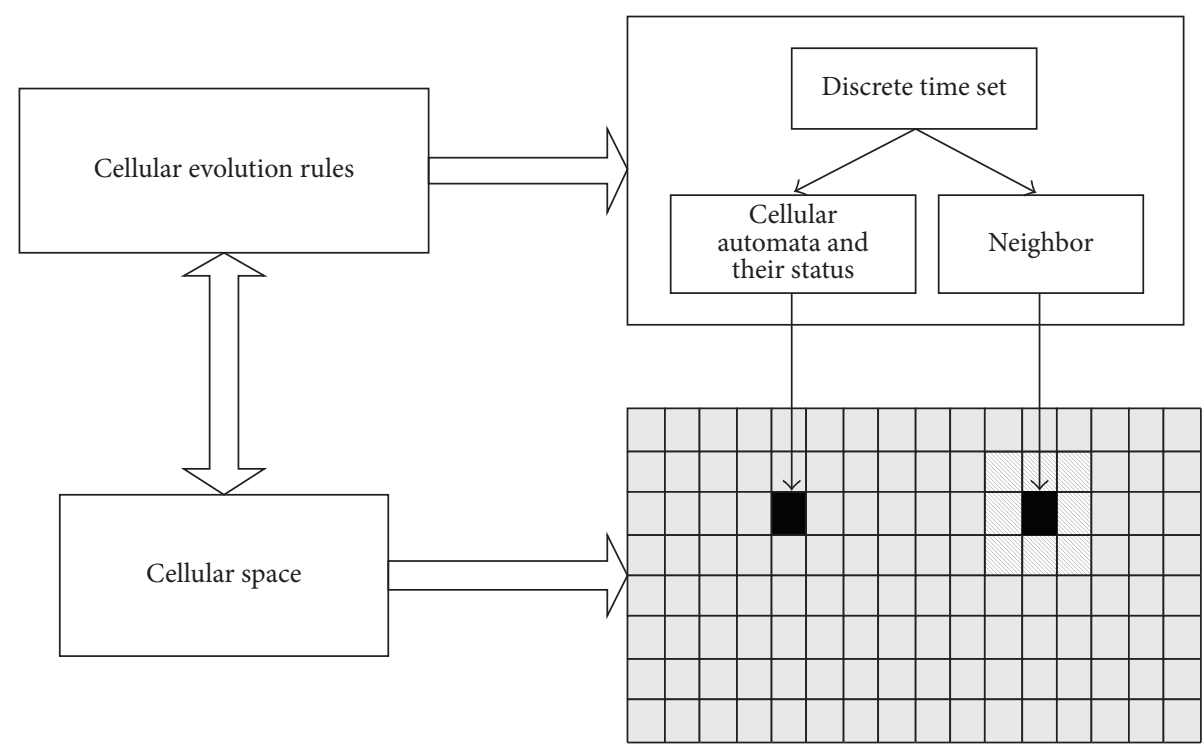

Figure 1: CA Composition.

\section{Evolution Model Building}

3.1. The CA Model. CA is composed of cellular space, cell (lattice), neighbor, cellular evolution rules, and finite cell state $\left(S_{1}, S_{2}, \ldots, S_{k}\right)$ (Figure 1).

$$
A=\left(L_{d}, S, N, f\right) .
$$

In the above formula [4], $A$ is CA system (cell is the basic unit of CA and is the research object of CA system). $L_{d}$ is cellular space ( $d$ is space dimension) and is the collection of cellular distribution space. $S$ is the set of cellular automata finite discrete state. $N$ is the set of neighbor cells which affect the next time state of the central cell. $f$ is the cellular state transition function, which represents the central cell state evolution rule from the current time to the next [5].

3.2. Bass Model Assumptions. According to the BASS model and the purpose of the simulation, we need to make the following assumptions.

(1) After the crisis, the information from the crisis includes the real information and the false information (rumor), and the other information may not be discussed [6]. Media and the public are likely to become the two kinds of information dissemination. The media and individuals have three possible states for two kinds of information; they are adoption, no adoption, and wait-and-see.

(2) $S$ indicates the above three states of the individual. $S=1$ indicates that the individual adopts the real information. $S=0$ indicates that the individual takes wait-and-see attitude to crisis information. $S=-1$ indicates that the individual adopts the rumors. The initial attitude of the individual to crisis information is decided by the individual's subjective consciousness.

(3) In the crisis information diffusion system, the largest scale of the total number of evolutionary individuals is certain, but the number of people who adopt crisis information is changing over time.

(4) In each evolution, the media and individuals have the right to change their own state. Media accepts the government's supervision and management and the people's supervision. So the attitude of the media to the crisis or the adoption state requires timely adjustment. For the public, its state is affected by three aspects [7]; one is the government, the second is the media, and the third is the surrounding neighbors.

(5) The state of the media is influenced by government's regulation and public reputation, and the state of the individual is influenced by the surrounding neighbors, the government, and the media. The degree of impact may not be the same, and it is represented by the weight.

(6) The government agency has the national credibility, so its attitude is generally cautious, and its state will not make regular adjustments. We consider that the state of the government is certain. In view of the government's aim of serving the people, it is assumed that the publicity information is accurate and true.

\subsection{Network Crisis Information Diffusion Model Building}

3.3.1. The Cellular Space $\left(L_{d}\right)$. It is assumed that cellular space is the whole system of crisis information diffusion. This model uses the $100 * 100$ model to construct the cellular space; row and column intersection represents the potential 
adopters of crisis information, so the number of individuals is involved in the evolution of the 10000 .

3.3.2. Cell. Each information demander or social individual is a cell. It is supposed that the whole diffusion system is divided into a set of square grids, each grid point representing an information need, and that it is a unit cell. A cell is a potential crisis information adopter; then we can use the ordered pair $(i, j)$ to express $(i=1,2, \ldots, 100 ; j=$ $1,2, \ldots, 100)$. It is the potential adopters' position in the cellular space, and the cellular $(i, j)$ represents the individual in the $j$ column of the $i$ column in the cellular space.

3.3.3. The Set of Cellular State. This model determines the set of cellular state $S=\{-1,0,1\}$. The three kinds of cellular state express the three chosen states in the face of the real (false) information. -1 indicates that the individual chooses to adopt the false information. 0 indicates that the individual is in a state of wait-and-see, and 1 indicates that the individual chooses to adopt the true information.

3.3.4. The Neighbor Form. A cell takes the cellular around it as its neighbor. In CA model, for two-dimensional cellular automata model, the most common is the Von Neumann type and the Moore type. Here we are using the Moore type. The state of the central individual or central element in the time $t+1$ is affected by the 8 neighbors around $t$. Cellular neighborhood is not the neighbor relationship in the model [8], and it is very complex. The distance between neighbors may be very far away, but the existence of the network can reduce or even eliminate the information lag distance.

3.4. Evolution Function and Cellular Evolution Rules. The state of a cell in the time $t+1$ depends on its own and its surrounding neighbors' state and the corresponding transformation rules.

$$
S_{i j}^{t+1}=F\left(S_{i j}^{t}, \Omega_{i j}, R\right) .
$$

$S_{i j}^{t+1}$ and $S_{i j}^{t}$ refer to the state of the $t+1$ moment and the $t$ moment in the position of $i j, \Omega_{i j}$ refers to the set of the surrounding neighbors' state collection, and $R$ refers to a series of transformation rules.

Whether individual transform adoption state into no adoption is affected by the internal and external factors. The mathematics expression is as follows:

$$
f(t+1)=I(t+1)+O(t+1) .
$$

In this formula, $I(t+1)$ is the internal factors. We assumes that individuals involved in the evolution have the ability to obtain information. So we should only consider the information needs of individual, and the individual information needs is the information requirements of individual expresses (C).

Compared with the internal factors, external factors are expressed as $O(t+1)$. We select the choice of information from the government credibility $(G)$, the media influence
TABLE 1: Transformation rules table.

\begin{tabular}{lcc}
\hline $\begin{array}{l}\text { State before the } \\
\text { conversion }\end{array}$ & Transformation rules & $\begin{array}{c}\text { The transformed } \\
\text { state }\end{array}$ \\
\hline$S(t)=-1$ & $0<p_{i j}^{t+1} \leq 0.2$ & $S(t)=-1$ \\
& $0.2<p_{i j}^{t+1} \leq 0.6[10]$ & $S(t)=0$ \\
& $0.6<p_{i j}^{t+1} \leq 1$ & $S(t)=1$ \\
\hline & $0<p_{i j}^{t+1} \leq 0.2$ & $S(t)=-1$ \\
$S(t)=0$ & $0.2<p_{i j}^{t+1} \leq 0.6[10]$ & $S(t)=0$ \\
& $0.6<p_{i j}^{t+1} \leq 1$ & $S(t)=1$ \\
\hline & $0<p_{i j}^{t+1} \leq 0.2$ & $S(t)=-1$ \\
$S(t)=1$ & $0.2<p_{i j}^{t+1} \leq 0.6[10]$ & $S(t)=0$ \\
& $0.6<p_{i j}^{t+1} \leq 1$ & $S(t)=1$ \\
\hline
\end{tabular}

$(E)$, and other people around individual (8 neighbors) as external influence factors [9]. Individual state transformation probability [10] is

$$
\begin{aligned}
p_{i j}^{t+1} & =\frac{f(t+1)}{\max (f(t+1))} \\
& =\frac{G(t+1)+E(t+1)+C(t+1)+V(t)}{\max (G(t+1)+E(t+1)+C(t+1)+V(t))} .
\end{aligned}
$$

Individual adoption behavior is influenced by the society (government credibility, media influence) and individual cognition of crisis information (information needs, neighbors' state). In the information adoption behavior model (IABM), there is positive correlation between individual adoption behavior and social influence, individual information cognition, and the influence degree from social influence; individual information cognition to individual adoption behavior is 0.6 . That is to say, when the factors want to change individual adoption behavior, the individual state transition probability should reach 0.6. Accordingly, when the individual state transition probability is not up to 0.6 , individual state is in a wait or not to adopt, and when the individual state transition probability is higher than 0.6 , the adoption state will change. Wait state is the transition state of adoption of different information, and when the individual state transition probability is between 0.2 and 0.6 , individual state is in a wait, and when the individual state transition probability is not up to 0.2 , individual state is not to adopt. Thus the individual state transition probability is divided into three categories, and the transformation rules are as shown in Table 1.

\section{The Simulation and the Analysis of the Results}

According to the above transformation rules, we suppose the initial state of the cellular is set to 0 , and set the number of potentially affected people is $N=10000$.

In the evolution model, the initial state of cellular is assigned randomly the values 1 and 0 according to the proportion of $1: 100$. That is to say, in the early days of crisis, there was only the wait-and-see crowd and a small number of 


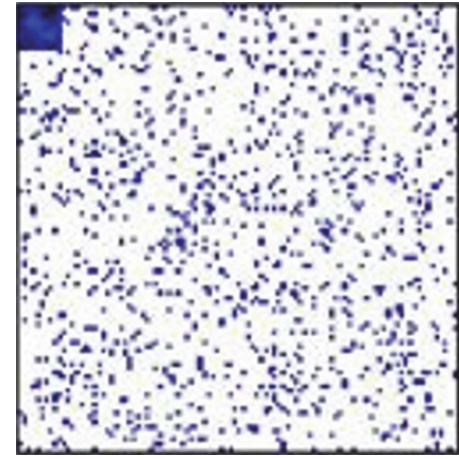

$t=10$

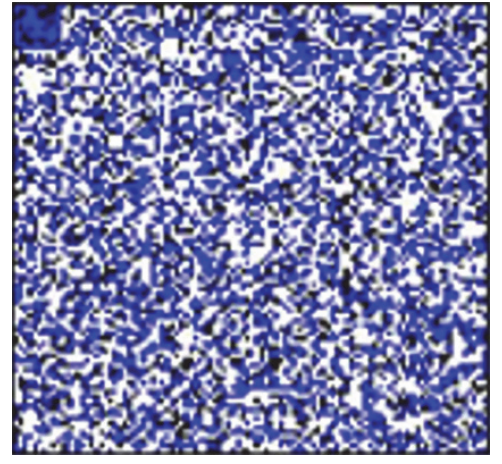

$t=30$

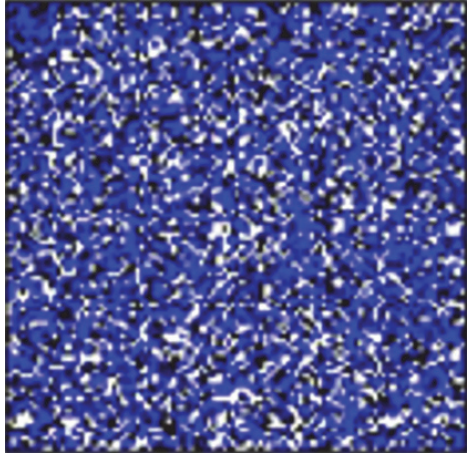

$t=100$

FIGURE 2: The initial evolution.

people who knew the actual information. And the emergence of the rumors is delay in the time; then trust rumors crowd in the process of evolution appear. In the event of a crisis, there is difference in number between the wait-and-see people and the people who know the truth. We simulate the influence on the effect of crisis information diffusion from the different initial state value of individual information needs $C$, the government's credibility $G$, and the media influence $E$. Each simulation runs 100 times.

After the initial value is set, its evolution process is shown in Figure 2. White represents the potential adopters of crisis information, the blue represents the true information adopters, and the black represents the false information adopters (the evolution graphics when $t=10, t=30$, and $t=100)$.

\subsection{The Influence of the Degree of Individual Information} Demand on the Diffusion Process of Crisis Information. Assuming that the values of the government credibility and media influence are set, we simulate the crisis information diffusion process by changing the degree of individual crisis information demand $C$. This is the crisis information adoption process of the individual. Because the influence of diffusion effect is mainly caused by the adoption behavior, so the whole evolution process is only applicable to the adoption crowd, and the wait-and-see state is only the excessive process of adoption. The evolution simulations run five times. We set the government credibility $G=0.5$ and the media influence $I=0.5$ which remain unchanged. That is to say, the government credibility and the media influence are in the stable medium level. And the values of individual information demand $C$ are $0.1,0.3,0.5,0.7$, and 0.9. According to the different values of individual information demand $C$, the total number of individuals who adopt the crisis information changes, as shown in Figure 3.

It is assumed that the government credibility and the media influence are at a moderate level.

(a) When $C=0.1$, the individual information need is average distribution between 0 and 0.1 . And when the degree of information of individual demand is low, the number of people impacted by crisis information is low. Then the majority of people are in the waitand-see state, and they are not concerned about or interested in the crisis information.

(b) When the value of $C$ is from 0.3 to 0.5 and from 0.5 to 0.7 , the changing of the number of three kinds users shows that the number of the wait-and-see people is declining with the rising of individual information demand, and the number of the adopters is increasing. This shows that the individual's adoption of crisis information will increase with the increase of the information demand. In addition, when $C=$ 0.9 , when the individual has a strong demand for information, the evolution results show that the final number of the affected people reaches $90 \%$. When the public is very concerned about the crisis, the number of the affected people is relatively more.

4.2. The Influence of the Government Credibility on the Diffusion Process of Crisis Information. Assuming that the values of the degree of individual crisis information demand $C$ and the media influence $E$ are set, we simulate the trend of individual choice to adopt crisis information by changing the government credibility $G$. We set the degree of individual crisis information demand $C=0.5$ and the media influence $E=0.5$. And the values of the government credibility $G$ are $0.1,0.3,0.5,0.7$, and 0.9 [11]. According to the different values of $G$, the number of individuals who choose to adopt true and false information is changing as shown in Figure 4.

By observing the changes of the number, we assume that the individual crisis information demand and the media influence keep stable.

(a) During the period of crisis information diffusion, when $G=0.1$, that is to say that the credibility of the government is low, and we find that a small number of people who adopt real information begin rising after the evolution of 100 times. Then the false information appears; the number of people who adopt the false information is more and more in the case of less intervention by authoritative organizations, even more than the number of people who adopt the real information. Because of the lack of authoritative 

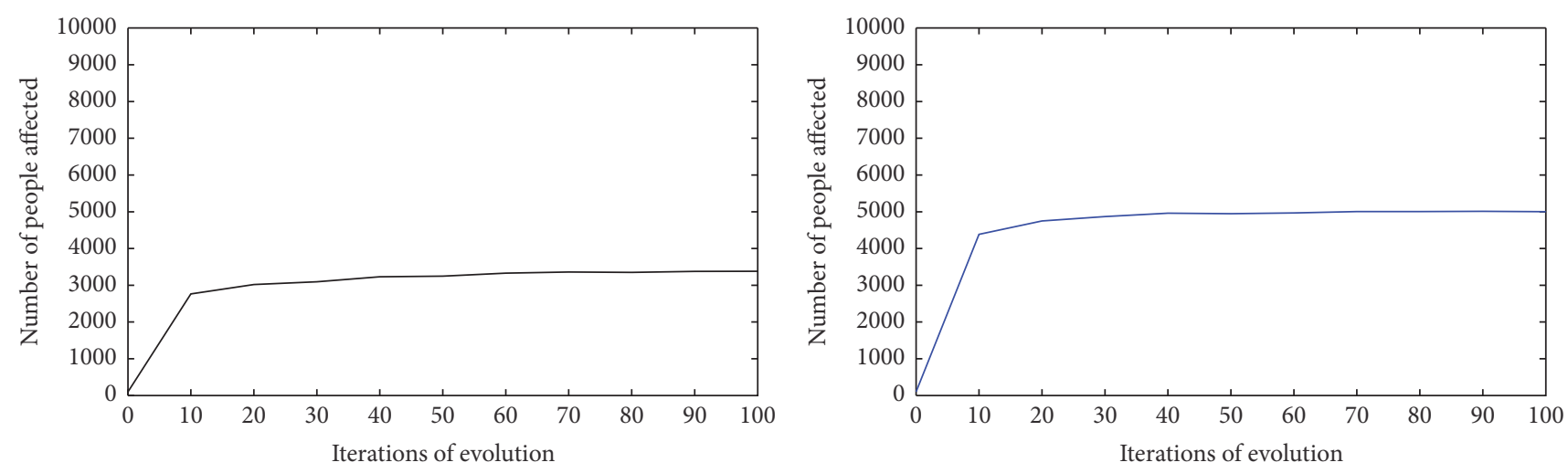

$$
C=0.1
$$

$$
C=0.3
$$
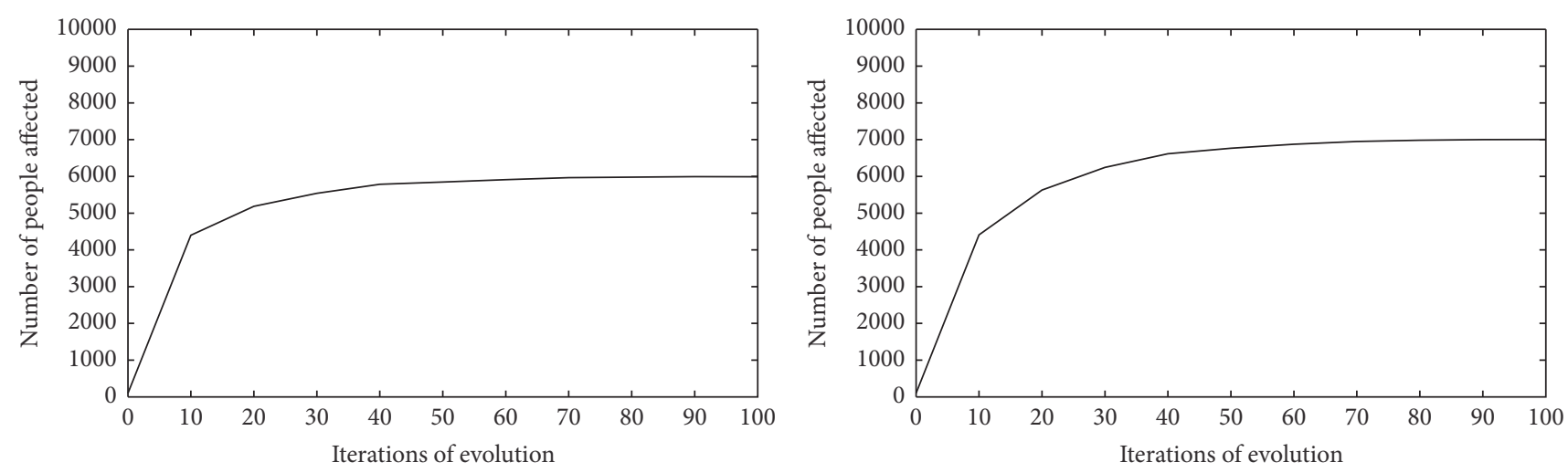

$$
C=0.5
$$$$
C=0.7
$$

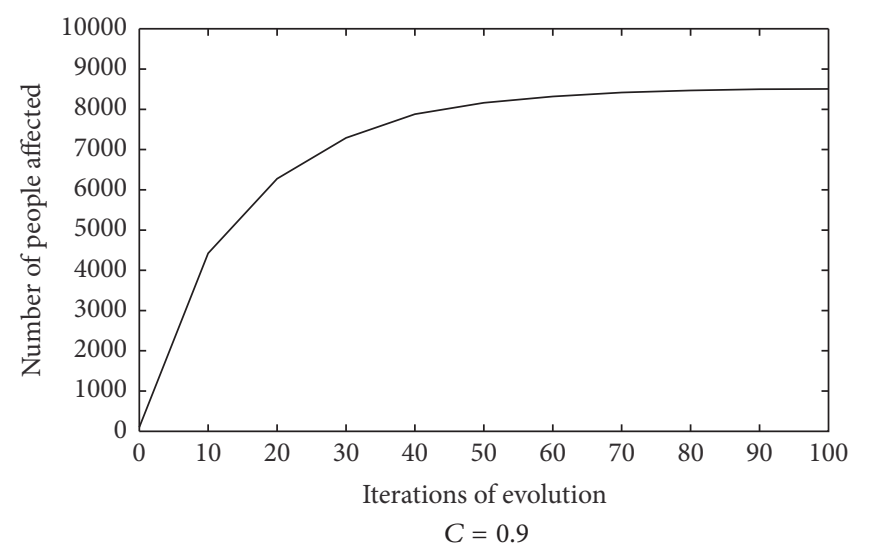

FIGURE 3: The total number of individuals to adopt information.

information on the release and support, the number of people to adopt the real information increases is decreasing, while the number of adopters to rumors is increasing, and the upward momentum is obvious. This shows that the number of people to adopt the real information is more than the number of people to adopt rumors; if the government takes the ignore attitude, it may lead to the rapid increase of the number of rumors. When the number of adopter to rumors is more than the number of adopter to the real information at a certain time, this will cause the great panic and form the unpredictable hazards. (b) When the value of the government credibility is from 0.1 up to 0.3 , it can be seen that the number of people to adopt the real information and the number of people to adopt the rumors are increasing and tend to be stable. The government intervention and propaganda play a key role in inhibiting the rumors, and the number of adopter to the real information in the diffusion process is more than the number of adopter to rumors. With $G$ increasing from 0.3 to 0.5 , $0.7,0.9$, the quantity gap between the adopter to the real information and the adopter to rumors is widening. It shows that the government information release 

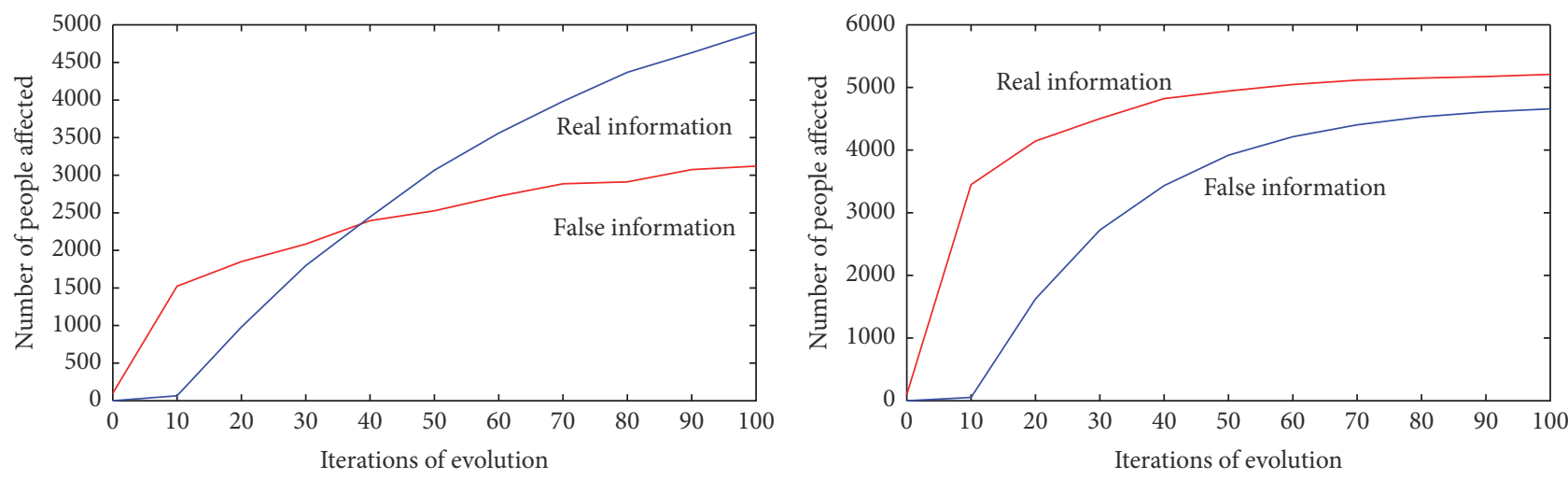

$G=0.1$
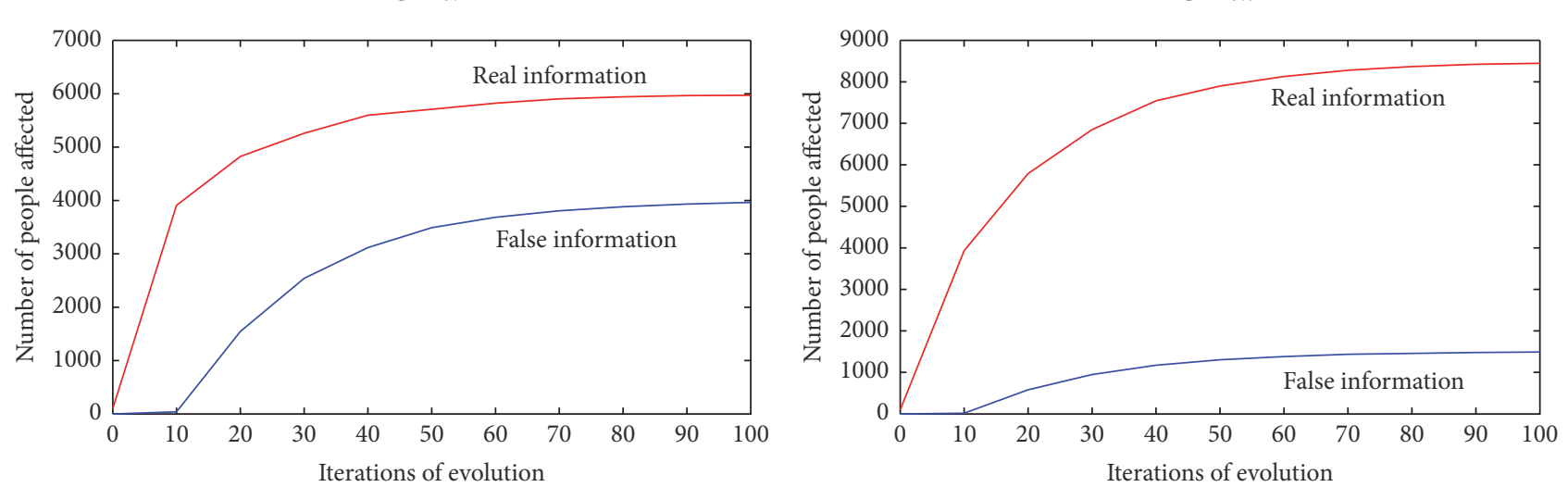

$$
G=0.5
$$

$$
G=0.7
$$

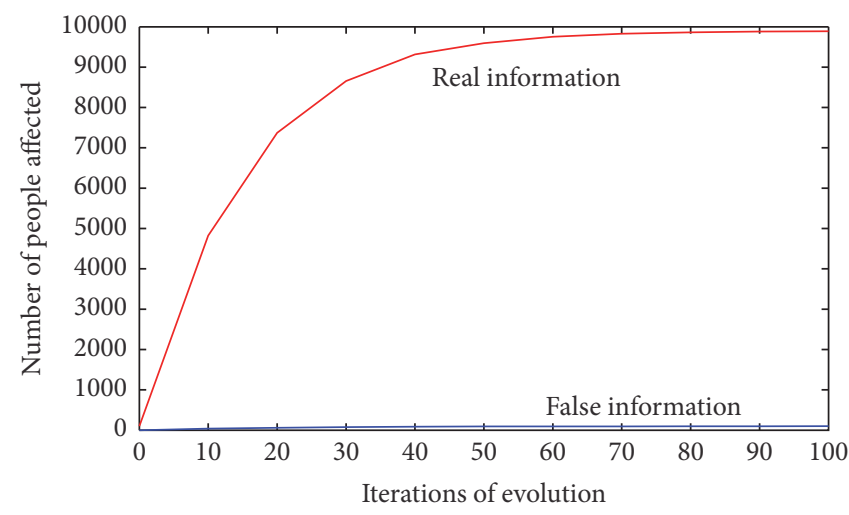

$G=0.9$

FIGURE 4: The number of individuals to adopt the real or false information.

behavior makes the wait-and-see people gradually trust the true information of the authority, which greatly inhibited the rumors spread and diffusion.

\subsection{The Influence of the Media Influence on the Diffusion} Process of Crisis Information. Assuming that the values of the degree of individual crisis information demand $C$ and the government credibility $G$ are set, we simulate the trend of individual choice to adopt crisis information by changing the media influence $E$. Because the media influence is supervised by the public opinion and the government, so the media's impetus to the information is built on the basis of the former two. We set individual crisis information demand $C=0.5$ and the government credibility $G=0.5$. In Figure 5 , when the government credibility $G$ and the media influence $E$ are 0.5 , the extent of their impact has been great. Taking the upper limit of $E=0.9$ [12], we can see that the media influence is under the government credibility, strengthening the propaganda. We should strengthen publicity; then the media influence can have a driving effect on the crisis information diffusion [13]. 


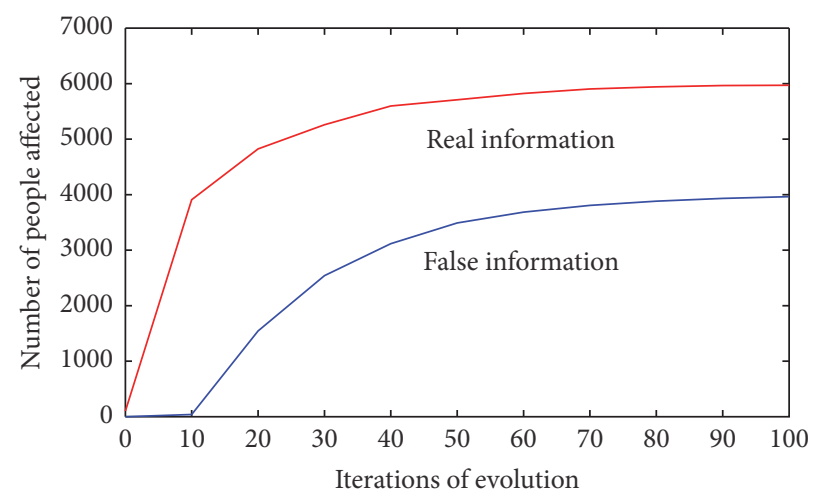

$E=0.5$

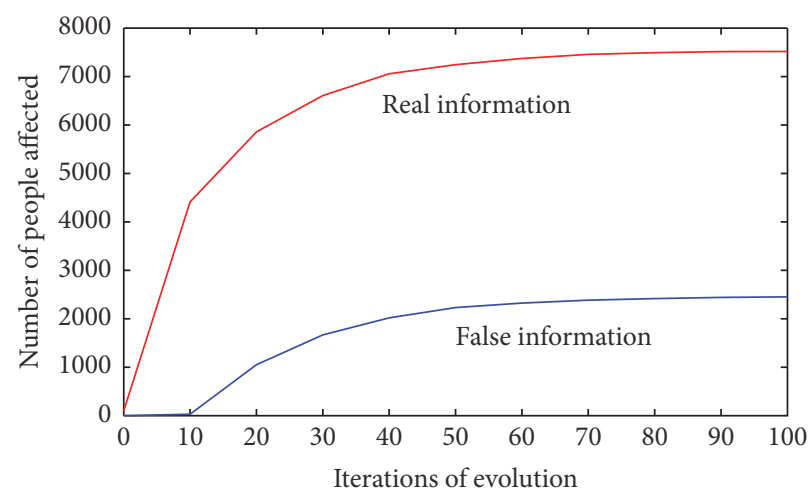

$E=0.9$

FIGURE 5: Different values of $E$ have effect on crisis information diffusion.

\section{Countermeasures}

5.1. Strengthen the Publicity of Government and Other Authoritative Organizations and Media. The government's response to the crisis information diffusion, the government's intervention, and the release of real information are necessary and critical, and the government's propaganda time should be judged according to the actual situation. The publicity of the government will help the public to grasp the real information and guide the network information. When the crisis information is mainly the real information, the government may conduct propaganda in a later time or not conduct propaganda. And when the crisis information is mainly the false information, the government's guidance has a key guiding role on the diffusion result. After the crisis, the crisis information will appear in the network at the first time. At this time, the government should use the Internet platform to publish relevant information of public crisis through official channels or media platform [14], in order to avoid the rapid spread of the false information.

5.2. Strengthen the Supervision of the Diffusion Channels. "The government cannot develop without credit," this is to say that the public trust is essential to the government construction. This requires that the government should establish the relevant accountability mechanisms and severely punish criminals who diffuse false information on the Internet platform to create panic and then increase the intensity of punishment. For the crisis information diffusion, the government should actively guide and strengthen supervision and then make the information diffusion in the direction of the good communication. The government should formulate the basic guidance and supervision strategy of public crisis information diffusion network. Also the government should dynamically adjust according to the actual situation of information diffusion and sum up the coping strategy according to the implementation results. The government has accumulated the experience to deal with the public crisis information diffusion in the network, in order to improve the coping ability of network information.
5.3. Improve the Accuracy and Authority of Information. When the media influence of the regular diffusion becomes larger, the number of the right information adopters is increasing. Therefore, during the crisis, expanding the coverage of the official formal media channels and speeding up the correct information transmission speed can effectively curb false information spread. At the same time, the extension of the real information dissemination scale can improve the accuracy of information. We should establish a healthy and orderly information release mechanism, improve the accuracy and the authority of the information, standardize information dissemination form, combined with influential media organizations to release information, and then spread scale accurate information. Also, we should strengthen the supervision of public opinion and reduce the distortion of the crisis information in the diffusion process.

5.4. Strengthen Public Emergency Quality Education. In the initial state of the crisis information diffusion, the number of people who adopt the real information and the rumors in the crisis information is closely related to the information diffusion effect. We can improve public emergency quality education by spreading the knowledge of all kinds of crisis and popularizing all kinds of crisis management knowledge. On the one hand, it can reduce the number of manufacturing rumors and solve the problem from the source. On the other hand, it can make the potential impact population increase the ability to distinguish and deal with rumors, in order to suppress the spread of rumors.

\section{Conclusion}

After the crisis, the crisis information will spread and diffuse through a variety of channels, and the information which eventually reaches the public will have the right information and the rumors. On the basis of the CA model, this paper constructs the diffusion model of the correct information and the rumor information and analyzes the difference of the diffusion regulation and the diffusion state of the two kinds of information under different parameters. The 
research results show that the factor affecting the scale of the crisis information diffusion is mainly the public's demand for information, and the two kinds of information diffusion are mainly influenced by the government credibility and the media influence. Based on this, this paper puts forward the countermeasures. This paper makes simulation analysis about the two types of crisis information diffusion in different parameters and how to combine the actual problem to further optimize the model is the follow-up research work.

\section{Competing Interests}

The authors declare that there are no competing interests regarding the publication of this paper.

\section{Acknowledgments}

This work was supported by the National Natural Science Foundation of China (71271062).

\section{References}

[1] M. E. Newman, "The structure and function of complex networks," SIAM Review, vol. 45, no. 2, pp. 167-256, 2003.

[2] F. M. Nicosia, "Some reflections on a study of diffusion of information," Journal of the Operational Research Society, vol. 16, no. 3, pp. 382-385, 1965.

[3] S. A. Delre, W. Jager, T. H. A. Bijmolt, and M. A. Janssen, "Will it spread or not? the effects of social influences and network topology on innovation diffusion," Journal of Product Innovation Management, vol. 27, no. 2, pp. 267-282, 2010.

[4] X. Dang, C. Zhang, and H. Zhanjun, "The research on network public opinion propagation model based on fuzzy cellular automata," Computer Engineering, vol. 40, no. 4, pp. 209-213, 2014.

[5] L. Fang, X. Gong, and R. Xiao, "Cellular automaton simulation of technological innovation diffusion," System Simulation Technology, vol. 3, no. 2, pp. 82-89, 2007.

[6] J. Wei, L. Zhou, and D. Zhao, "Crisis information diffusion model based on BASS model," System Engineering, vol. 29, no. 9, pp. 20-22, 2011.

[7] Z. Song, F. Qiao, and L. Shi, "The research on the diffusion of emergent events rumor information based on BASS model," Intelligence Magazine, vol. 35, no. 1, pp. 100-104, 2016.

[8] R. Baskerville and J. Pries-Heje, "Diversity in modeling diffusion of information technology," Journal of Technology Transfer, vol. 28, no. 3-4, pp. 251-264, 2003.

[9] S. Lim, I. Jung, S. Lee, and K. Jung, "Analysis of information diffusion for threshold models on arbitrary networks," The European Physical Journal B, vol. 88, pp. 201-215, 2015.

[10] X. Song, Research on Construction and Application of User Information Adoption Behavior Model, Jilin University, Changchun, China, 2010.

[11] P. S. Van Eck, W. Jager, and P. S. H. Leeflang, "Opinion leaders' role in innovation diffusion: a simulation study," Journal of Product Innovation Management, vol. 28, no. 2, pp. 187-203, 2011.

[12] L. Ren, Z. Liang, Z. Du, and Y. Li, “The research review of network public opinion evolution model on complex networks," Intelligence Science, vol. 32, no. 8, pp. 148-156, 2014.
[13] Q. Zhong, Q. Wei, and L. Zhang, "The social crisis information diffusion model under the Lotka-Volterra system," Systems Engineering Theory and Practice, vol. 32, no. 1, pp. 104-110, 2012.

[14] C. Xiaojian, Z. Liu, and F. Zeng, "The control research on public crisis information dissemination network based on the theory of the small world," Intelligence Theory and Practice, vol. 33, no. 5, pp. 80-84, 2010. 

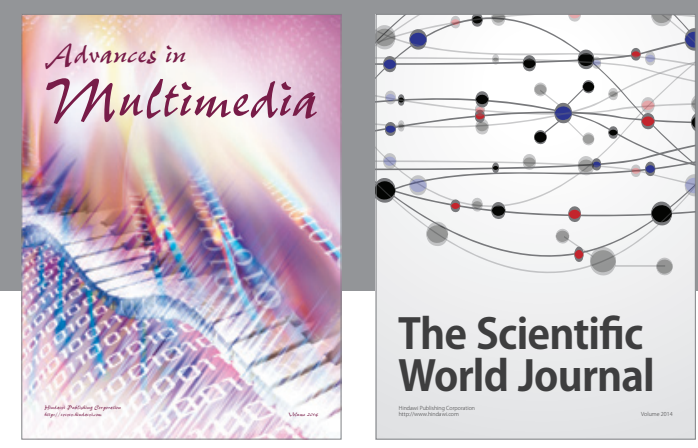

The Scientific World Journal
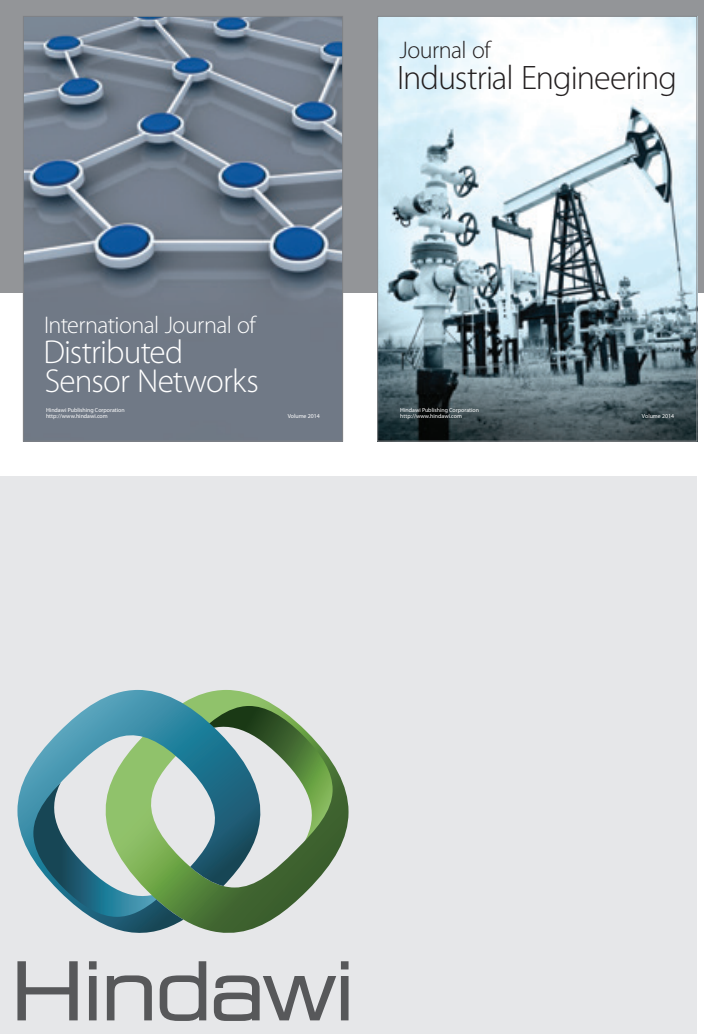

Submit your manuscripts at

http://www.hindawi.com

\section{Computer Networks} and Communications
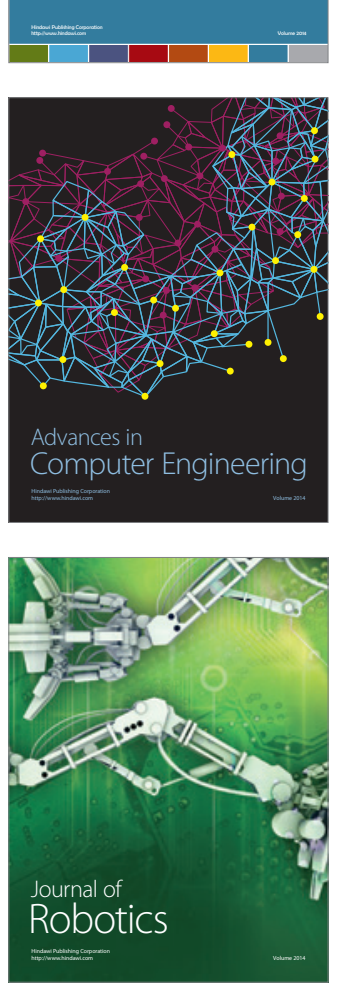
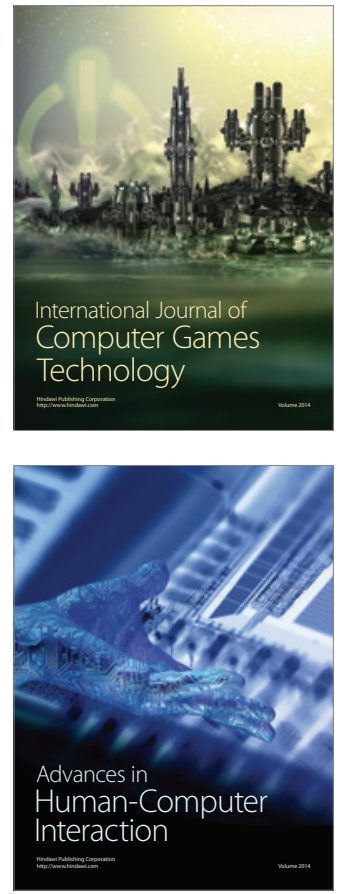
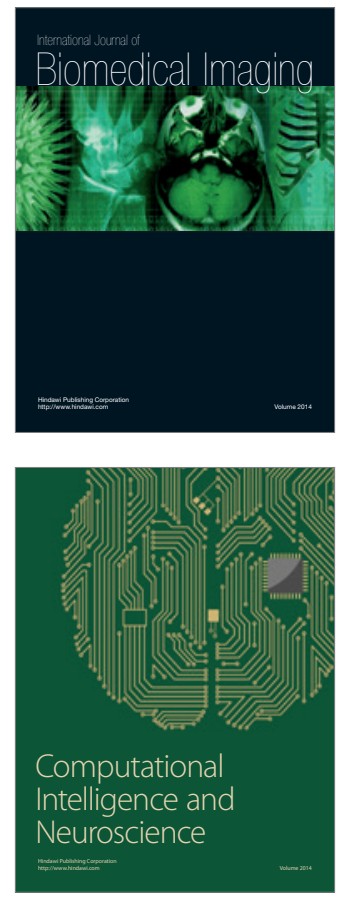
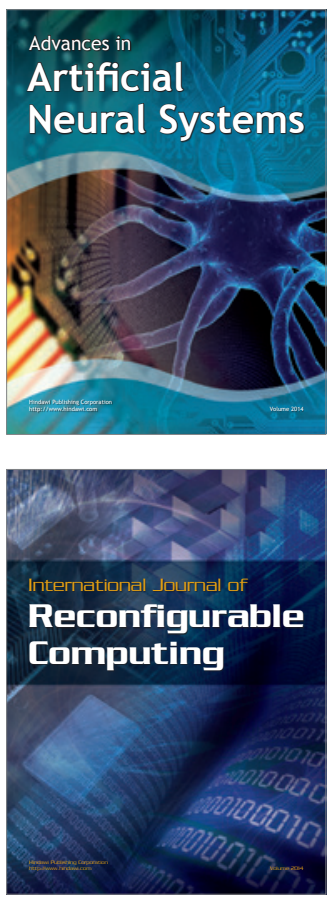
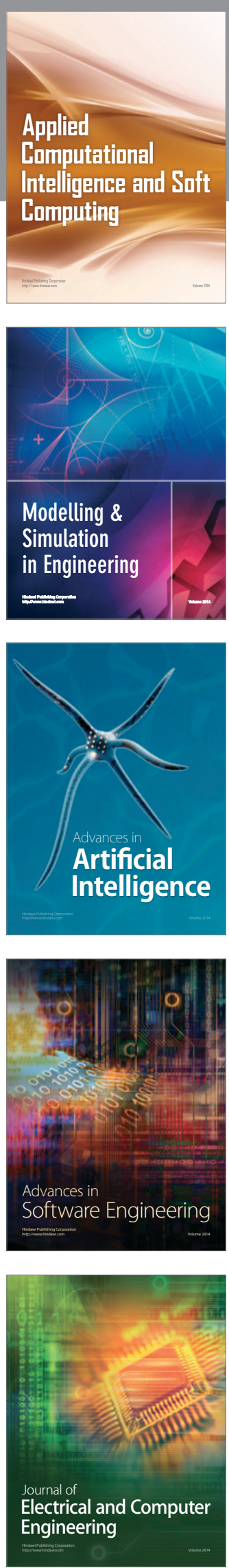